\title{
Exposition sociocognitive et évaluation des risques : le cas de la téléphonie mobile
}

\author{
M. POUMADÈRE ${ }^{1}$, A. PERRIN ${ }^{2}$
}

(Manuscrit reçu le 9 juin 2010, accepté le 31 octobre 2010)

\begin{abstract}
RÉSUMÉ La téléphonie mobile se caractérise par un développement mondial spectaculaire. En corollaire, les radiofréquences deviennent omniprésentes dans l'environnement privé et public : à la fois par la technologie nécessaire au fonctionnement de la téléphonie mobile et par la prise de conscience par le plus grand nombre de leur existence. Cette dualité se traduit par l'inclusion des préoccupations des populations dans l'évaluation des risques. Dans ce contexte, nous examinons d'abord le principe de séparation entre évaluation et gestion des risques. Ensuite, nous considérons plusieurs catégories d'exposition. Le concept d'exposition sociocognitive est ainsi avancé à titre exploratoire, pour prendre en compte les éventuels effets d'une exposition chronique des populations à des informations préoccupantes quand diverses conséquences sanitaires des radiofréquences sont évoquées. Cette approche précise la place de l'information comme variable intermédiaire dans la relation entre environnement et santé. Appliquée au cas des radiofréquences, cette approche pourrait conduire à redéfinir les populations vulnérables, les situations extrêmes et les mesures de protection.
\end{abstract}

ABSTRACT Sociocognitive exposure and risk assessment: The case of mobile phones.

Mobile telephone technology is characterized by spectacular global expansion. In a corollary manner, radio frequencies have become omnipresent in the public and private environment, as the physical basis for mobile communications, and as something that has entered the awareness of a vast number of persons. This dual nature of radio frequencies means that population concerns have been taken into account in the risk assessment process. Against this background, we first examine the principle of separation between assessment, evaluation and management of risk. We then consider several categories of exposure. The concept of sociocognitive exposure is proposed, to address the possible effects of chronic exposure of populations to alarming information when various health effects of radio frequencies are discussed. This approach specifies the role of information as an intermediary between environment and health. Applied to the case of radio frequencies, such a conceptual approach could result in redefining such terms as vulnerable populations, extreme situations and protective measures.

Key words: Radio frequencies / awareness / information / sociocognitive exposure

\footnotetext{
1 Institut Symlog, Gouvernance des risques, 262 rue Saint-Jacques, 75005 Paris, France.

2 Institut de recherches biomédicales des armées, CRSSA Grenoble, Département de radiobiologie, 24 avenue des Maquis du Grésivaudan, 38700 La Tronche, France.
} 


\section{Introduction générale}

En contraste avec les nombreuses évaluations de risques conduites de façon routinière et servant la décision en gestion et communication des risques, certaines situations paraissent se distinguer. Ainsi les radiofréquences font-elles l'objet d'une attention soutenue, cependant que leur dangerosité n'a pas été établie malgré la répétition d'études. Cette situation trouve son origine en grande partie dans l'engouement pour la téléphonie mobile qui les a rendues omniprésentes dans l'environnement privé et public. Quand l'évaluation des risques porte sur un objet dont les dimensions psychologiques et sociales constituent des caractéristiques déterminantes, se posent les questions théoriques et méthodologiques de l'intégration de ces dimensions dans l'évaluation. En effet, l'évaluation du risque pour ce type d'objet nous met face au dilemme présenté par l'alternative suivante : ignorer la dimension sociale des objets à évaluer (et délaisser des préoccupations dont l'expression est légitime dans un système démocratique) ou les prendre en compte (en risquant de remettre en cause la séparation entre évaluation et gestion des risques). Pour dépasser ce dilemme, nous proposons d'étendre la notion d'exposition: alors que la recherche de relations causales entre exposition aux ondes et effets biologiques ou sanitaires constitue l'objet principal de l'expologie, nous proposons de recourir, en complément et à titre exploratoire, à la notion encore peu étudiée d'exposition sociocognitive. L'objectif ici étant de retenir le fait que les populations sont exposées non seulement aux radiofréquences en tant qu'agent physique mais aussi à la téléphonie mobile en tant qu'objet social. Diverses conséquences sanitaires des radiofréquences sont en effet évoquées et la question se pose des éventuels impacts d'une exposition chronique à des informations préoccupantes.

\section{Cadrages de l'évaluation des risques et téléphonie mobile}

L'évaluation des risques (dans le sens de risk assessment) repose sur l'identification d'un danger pour la santé, tel que peuvent le révéler, par exemple, les études biologiques et/ou épidémiologiques. Elle se donne pour objectif d'estimer la gravité et la vraisemblance d'une atteinte à la santé ou à l'environnement, résultant de l'exposition à un agent ou à une activité susceptible de causer des dommages sanitaires et environnementaux. Cette définition se retrouve implicitement dans la mission qui est confiée à l'expertise de $l^{\prime}$ Afsset ${ }^{3}$ concernant les radiofréquences.

\footnotetext{
3 Ayant participé à l'expertise collective de l'Afsset (Agence française de sécurité sanitaire de l'environnement et du travail) se rapportant aux risques sanitaires des radiofréquences (Afsset, 2009), les auteurs sont redevables de ce cadre de travail et des échanges fructueux avec les autres experts. Les points de vue présentés sont ceux des auteurs et n'engagent que leur seule responsabilité.
} 
Ainsi, la saisine 4 à l'origine du rapport «Radiofréquences » paru en 2009 demandait-elle d'accorder «une importance toute particulière à l'étude des " signaux » que l'Agence avait identifiés dans son précédent avis (modification éventuelle de la perméabilité de la barrière hémato-encéphalique, étude épidémiologique ayant montré une possible augmentation du risque de neurinome de l'acoustique chez les personnes ayant utilisé longtemps certains types de téléphones mobiles) (...), notamment en termes d'exposition du public ». Il était en outre demandé «d'identifier avec la plus grande attention les préoccupations de la société civile et de contribuer ainsi au débat public sur ce thème », soulignant ainsi la dimension sociale du risque. Le dispositif d'expertise construit par l'Afsset a donc réuni plusieurs disciplines recouvrant les sciences des domaines biomédical et physique, ainsi que les sciences humaines et sociales (SHS).

Dans le cas d'expositions à faibles doses, le travail d'identification des effets biologiques et sanitaires pour évaluer le risque revient souvent à estimer le degré d'incertitude résiduel. Ce travail s'effectue à partir de l'état des connaissances, fondé sur l'analyse de l'ensemble des données de la littérature scientifique retranscrite dans des rapports d'expertises collectives. C'est le cas pour les expositions aux radiofréquences utilisées par la téléphonie mobile dont des effets éventuels sont recherchés à des niveaux d'exposition inférieurs aux seuils réglementaires en vigueur. Ces derniers sont établis en fonction des effets connus à ce jour qui apparaissent en réponse à l'échauffement causé par l'exposition radiofréquence à partir d'une certaine puissance d'émission (ICNIRP, 1998). La réglementation impose une marge de sécurité visant à prendre en compte les situations extrêmes et les populations plus vulnérables pouvant être rencontrées.

Parallèlement, les recherches en SHS dans le domaine du risque portent généralement sur trois niveaux. Le premier concerne les méthodes et concepts permettant, en lien avec l'analyse des décisions, de diffuser et d'implémenter les actions de gestion des risques. Le deuxième niveau concerne l'organisation de la délibération collective et l'ingénierie du débat, associant les parties prenantes dans le cadre de la gouvernance des risques. Le troisième niveau est peut-être moins connu mais tout aussi important, puisqu'il s'agit de caractériser les objets et situations relevant du risque, en particulier par une analyse psychologique et sociale du rapport au risque, contribuant ainsi à son évaluation.

La saisine de l'Afsset demande donc de prêter attention au débat social, ainsi qu'aux préoccupations du public. Ce point est délicat dans la mesure où le principe

\footnotetext{
4 Saisine $n^{\circ} 2007 / 007$ « Mise à jour de l'expertise relative aux radiofréquences » émise le 14 août 2007 par le Ministère de la santé, de la jeunesse et des sports et par le Ministère de l'écologie, du développement et de l'aménagement durables, voir p. 457, rapport Afsset (2009).
} 
de séparation des différents niveaux de l'analyse des risques paraît remis en question. En effet, pour éviter que l'évaluation ne soit prédéterminée par le pouvoir politique, les enjeux économiques, l'amplification sociale du risque (Pidgeon et al., 2003), ou les pressions militantes, un principe tôt instauré en matière d'analyse des risques consiste à séparer l'évaluation d'un risque de sa gestion et de la communication s'y rapportant. Cela dans la mesure où la notion de risque inclut un danger potentiel dont on cherche à établir la nature, la plausibilité ainsi que la possibilité d'occurrence sur des fondements scientifiques. Cette séparation n'étant pas un état de nature, elle résulte de diverses réflexions et aménagements institutionnels entrepris vers la fin des années 1970. La création plus récente des Agences en France s'inscrit également dans cette perspective d'indépendance de l'expertise en évaluation des risques (Hubert, 2000). Ce cadre permet de s'écarter d'une vision fusionnelle de l'analyse des risques où les tâches et les rôles seraient comme indifférenciés, les interfaces (notamment celle entre science et politique) et les inévitables arbitrages demeurant hors de la pensée du risque. C'est donc l'existence d'une séparation, et d'acteurs distincts, qui permettent d'instaurer un dialogue entre évaluation et gestion des risques.

Notons que cette séparation entre les différentes composantes de l'analyse des risques n'exclut pas la possibilité d'engager des expertises pluralistes, reposant non seulement sur l'interdisciplinarité mais aussi sur la participation, par exemple, de représentants associatifs et d'acteurs impliqués. Il peut s'agir de membres de populations locales à la source du «signal», lequel peut ainsi être pris en compte sous cette forme également. Une autre situation à considérer est celle où la rapidité d'une innovation technologique, comme par exemple dans le domaine médical, produit de facto un bénéfice global favorable avant que la démarche d'optimisation en qualité et sécurité, dont le cycle est plus long, n'ait pu se déployer entièrement (Amalberti, 2009).

Pour intégrer dans l'évaluation du risque les éléments se rapportant au débat social et aux préoccupations du public, il nous apparaît important de considérer ces éléments non pas comme un produit de l'incertaine dangerosité des radiofréquences, mais comme une possible entrée parmi les facteurs pouvant se traduire par un impact sanitaire. Ce cadrage suppose une approche méthodologique spécifique et nous recourons à cet effet à la notion encore peu développée d'exposition sociocognitive.

\section{Notion d'exposition sociocognitive}

Les caractéristiques des radiofréquences en tant qu'agent physique, avec les éventuels effets biologiques qui leur seraient associés, constituent la première catégorie d'exposition pour l'évaluation des risques et un volume important de 


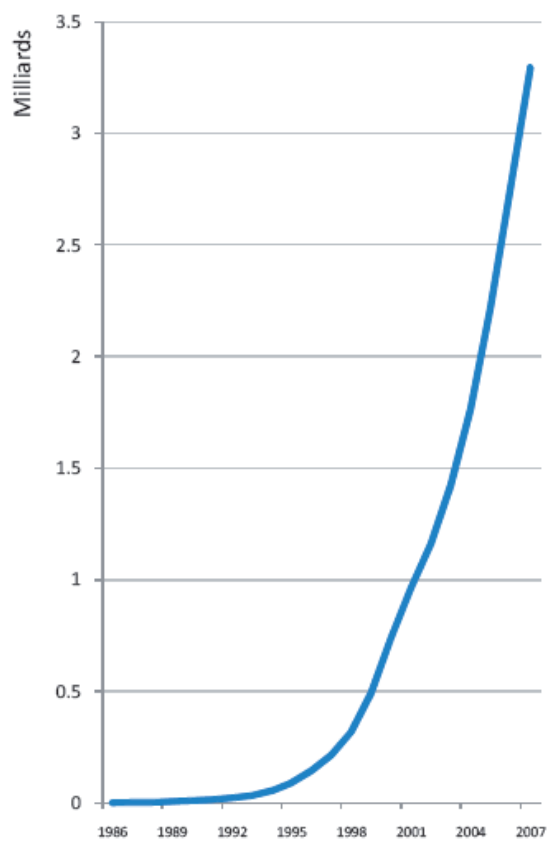

Figure 1 - Téléphonie mobile. Évolution du nombre d'abonnés dans le monde de 1985 à 2007 (OCDE, 2008).

Mobile phone. Evolution of the number of subscriptions in the world from 1985 to 2007 (OCDE, 2008).

résultats existe à ce niveau (Afsset, 2009 ; Veyret, 2010). Il s'agit également d'intégrer les caractéristiques de la téléphonie mobile en tant qu'objet social relativement nouveau mais très largement répandu dans l'environnement de chacun. En effet, la téléphonie mobile se caractérise par une diffusion massive, rapide et mondiale, le nombre d'abonnés passant de 750000 en 1985 à trois milliards en 2007 (voir Fig. 1) pour atteindre actuellement quelque cinq milliards. Les effets de l'exposition généralisée à cette nouvelle technologie et à son usage sont relativement peu connus et peuvent être à l'origine de troubles et préoccupations dans la population, compte tenu de possibles difficultés d'adaptation (Dab, 2010).

Cependant que ce développement exponentiel s'accompagne d'une couverture croissante du territoire par le réseau de télécommunication via les antennes relais, il existe de plus une troisième catégorie d'exposition correspondant aux nombreuses et diverses informations se rapportant aux risques et controverses dans le domaine des radiofréquences. La recherche en perception des risques a montré 
comment le rapport individuel et collectif au risque est en partie déterminé par l'information (Slovic, 2000 ; Renn, 2008).

Concernant les radiofréquences, un premier niveau d'information est procuré par la présence «matérielle» des agents physiques dans l'environnement. Cette relation est illustrée dans une étude où le radon et les lignes à haute tension sont traités comme deux cas de risques de rayonnements soulevant régulièrement divers niveaux d'attention parmi les populations et les experts (Poortinga et al., 2008). Ces cas sont proches sur plusieurs points des radiofréquences (en précisant que la dangerosité du radon est avérée), avec notamment l'absence chez l'homme d'un moyen de perception naturelle du rayonnement. L'étude montre que les croyances les concernant diffèrent en fonction du niveau d' « exposition visuelle » aux lignes à haute tension. Les auteurs concluent que ces résultats reflètent les informations visuelles dont disposaient les répondants «exposés », la prise de conscience (awareness) étant mesurée par la question suivante : "Pouvez-vous voir des lignes à haute tension depuis une ou plusieurs fenêtres ou portes de votre maison? ». Cette étude sur les effets de l'exposition visuelle est intéressante, dans la mesure où la mise en question et parfois le rejet des antennes relais commencent par leur identification visuelle.

Les technologies radiofréquences sont également associées à une importante production d'études cherchant à identifier des risques. Comme le montre la figure 2,

Peer Reviewed RF Bioeffects Publications

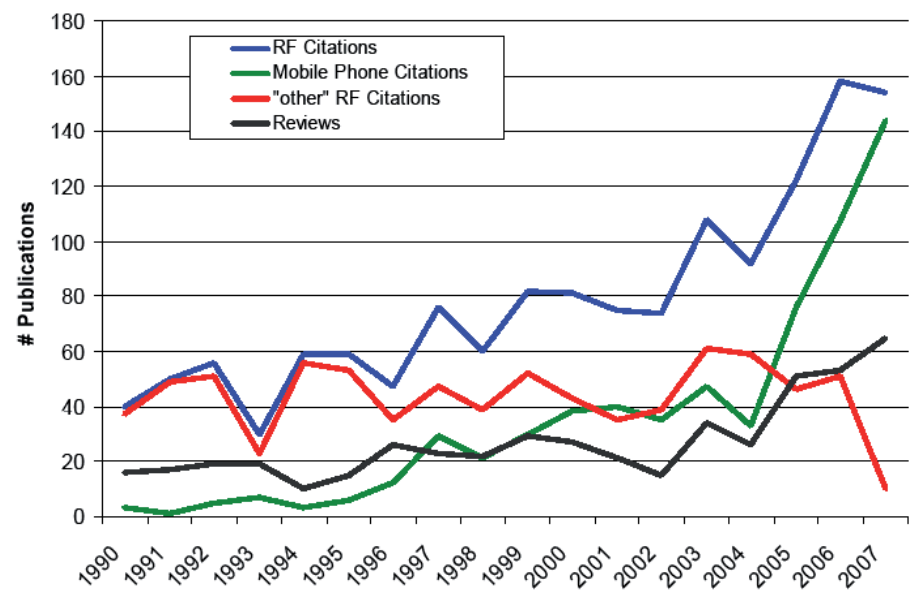

Figure 2 - Publications relatives aux effets biologiques des radiofréquences (Afsset, 2009).

Published research relating to biological effects of radiofrequencies (Afsset, 2009). 
les articles consacrés à la recherche d'effets biologiques des radiofréquences ont connu une progression rapide. Les citations concernant les radiofréquences depuis 1995 apparaissent prééminentes en comparaison avec les études consacrées à d'autres sources de radiofréquences, et celles concernant la téléphonie mobile montrent une très nette augmentation au cours des dernières années.

En rapprochant cette courbe de la précédente qui montre la diffusion de la téléphonie mobile dans le monde, on constate un développement parallèle entre cette diffusion et la production de publications. Que peut-on conclure de ce rapprochement ? Les savoirs scientifiques se rapportant aux radiofréquences (en tant qu'agent physique) n'étant pas vraiment nouveaux, il est vraisemblable que la multiplication des études sur les effets biologiques soit liée au développement de la téléphonie mobile, non seulement en tant que technologie, mais surtout en tant qu'objet social largement diffusé. Cela peut s'interpréter déjà comme une forme de réponse aux préoccupations et mobilisations des populations, illustrant la fonction sociale de la recherche et de l'évaluation des risques puisque la décision de les engager ne se limite pas à de seuls critères d'utilité scientifique (dont notamment l'acquisition de savoirs nouveaux).

Quant au contenu des études consacrées aux radiofréquences, le tableau I donne un aperçu des domaines étudiés lorsque des effets biologiques sont recherchés (première colonne) et lorsque des études épidémiologiques sont engagées pour rechercher des associations de pathologies avec l'exposition aux antennes relais et à aux téléphones mobiles (deuxième colonne). La troisième colonne présente une liste des symptômes attribués aux champs électromagnétiques par les personnes déclarant s'en trouver affectées. Ces listes se retrouvent en grande partie dans les rubriques sur lesquelles l'expertise de l'Afsset (2009) a porté et, sans forcément être exhaustives, montrent l'étendue et la complexité du sujet.

Comment ces objets d'études se transforment en information diffusée par les différents médias, portée à l'attention du public et, associée à d'autres représentations sociales, devient un sujet de préoccupations, est un domaine également complexe qui semble avoir été peu étudié. Une meilleure connaissance de ce processus devrait permettre de mieux comprendre les relations entre information sur les risques et impacts sur la santé. De plus, l'information ne se limite pas aux seules données de la recherche et il existe une grande diversité narrative dans les récits se rapportant à la téléphonie mobile ${ }^{5}$. On sait aussi que les médias et le public accordent le plus souvent leur attention aux conséquences

\footnotetext{
5 Par exemple, le journal espagnol Malaga Hoy présente le 24 novembre 2009 l'information suivante : « Les 350 habitants de Pérez sur la commune de Velez-Malaga, ont constaté 43 cas de cancers dont 35 ont entraîné la mort de riverains. Ils exigent le retrait des antennes relais ». www.malagahoy.es/article/provincia/571081/culpan/una/antena/telefonia/movil/casos/cancer.html
} 


\section{TABLEAU I}

Effets biologiques recherchés, sujets d'étude en épidémiologie et symptômes attribués aux radiofréquences.

Research biological and clinical effects, epidemiological topics, and symptoms attributed to radiofrequencies.

\begin{tabular}{|c|c|c|}
\hline $\begin{array}{l}\text { Effets biologiques et cliniques } \\
\text { (radiofréquences) }\end{array}$ & $\begin{array}{c}\text { Épidémiologie } \\
\text { (antennes-relais, téléphones } \\
\text { sans-fil) }\end{array}$ & $\begin{array}{l}\text { Symptômes attribués (exposition } \\
\text { à des champs électromagnétiques) }\end{array}$ \\
\hline $\begin{array}{l}\text { - Expression génique et synthèse de } \\
\text { protéines } \\
\text { - Stress oxydant et production de } \\
\text { radicaux libres } \\
\text { - Matériel génétique (ADN) } \\
\text { - Apoptose } \\
\text { - Développement de cancers chez } \\
\text { l'animal } \\
\text { - Système immunitaire } \\
\text { - Système nerveux } \\
\text { - Développement } \\
\text { - Reproduction } \\
\text { - Système auditif } \\
\text { - Système oculaire } \\
\text { - Système cardio-vasculaire } \\
\text { - Mélatonine } \\
\text { - Prolifération cellulaire } \\
\text { - Erythropoïèse chez le rat } \\
\text { - Cellules souches précurseurs chez } \\
\text { la souris } \\
\text { - Activité de l'ornithine } \\
\text { décarboxylase (ou ODC) } \\
\text { - Endocytose } \\
\text { - Cycle cellulaire }\end{array}$ & $\begin{array}{l}\text { - Agrégats de cas de cancers } \\
\text { (antennes-relais) } \\
\text { - Tendances temporelles } \\
\text { d'incidence et de mortalité des } \\
\text { tumeurs cérébrales } \\
\text { - Tumeurs cérébrales lié à la } \\
\text { proximité d'une station de base } \\
\text { DECT } \\
\text { - Gliomes } \\
\text { - Méningiomes } \\
\text { - Neurinomes du nerf acoustique } \\
\text { - Tumeurs des parotides } \\
\text { - Tumeurs de l'hypophyse } \\
\text { - Cancer des testicules } \\
\text { - Cancer du sein chez l'homme } \\
\text { - Mélanomes oculaires }\end{array}$ & $\begin{array}{l}\text { - Signes cutanés (face) } \\
\text { - Fatigue } \\
\text { - Palpitation } \\
\text { - Maux de tête } \\
\text { - Difficultés concentration } \\
\text { - Troubles du sommeil } \\
\text { - Nervosité } \\
\text { - Douleurs ostéo-musculaires } \\
\text { - Troubles respiratoires } \\
\text { - Troubles de l'équilibre } \\
\text { - Acouphènes } \\
\text { - Troubles de l'audition } \\
\text { - Troubles de la vision } \\
\text { - Signes cutanés (généraux) } \\
\text { - Engourdissement de la tête } \\
\text { - Troubles de la mémoire } \\
\text { - Irritation } \\
\text { - Agitation } \\
\text { - Spasmes intestinaux } \\
\text { - Jambes (agitation) }\end{array}$ \\
\hline
\end{tabular}

d'une éventuelle atteinte à la santé, délaissant les informations se rapportant, le cas échéant, à l'absence de causalité établie et au fait que le risque sanitaire ne soit pas avéré et peu plausible. Cela explique peut-être la relative rareté de responsables refusant publiquement de cultiver l'incertitude sur les radiofréquences pour privilégier explicitement la prévention d'autres risques ${ }^{6}$. Les études en perception des risques montrent qu'une majorité de personnes (en France comme dans la plupart des pays occidentaux) associent des risques pour la santé à la téléphonie mobile. Toutefois, ces perceptions ne se traduisent pas par un impact en termes de consommation et d'usage, lesquels continuent de se développer. Une explication simple peut être que les avantages apportés au plus grand nombre par la téléphonie

\footnotetext{
6 Exemple rare, L. Riley, directrice médicale au Massachussetts General Hospital, Boston, indique clairement que le port de vêtements anti-ondes par les femmes enceintes n'est pas la meilleure solution : « Au lieu de dépenser du temps et de l'argent à acheter une Belly Band, les femmes enceintes devraient s'employer à bien manger et à faire de l'exercice, ce qui est radical pour aider à réduire les effets négatifs d'une éclampsie ou d'un diabète gestationnel chez l'enfant à naître ». http://www.slate.fr/lien/27005/faut-il-porter-des-vetements-anti-ondes
} 
mobile l'emportent sur les risques perçus, bien que ces derniers paraissent relativement élevés à l'heure actuelle (Afsset, 2009).

La notion d'exposition sociocognitive renvoie donc au fait que les populations se trouvent exposées non seulement à un agent physique, comme dans le cas des radiofréquences, mais aussi aux nombreuses informations concernant la présence et les usages de la téléphonie mobile. Ce type d'exposition pose la question des relations entre environnement, information et santé.

\section{Liens entre environnement, information sur les risques et santé}

Les troubles et préoccupations associés aux radiofréquences figurent parmi les syndromes médicalement inexpliqués attribués à l'environnement, ou SYMIALE (Dab, 2010). Ainsi les personnes dites électrosensibles ou électrohypersensibles (EHS) attribuent-elles un certain nombre de symptômes atypiques, divers et variés, à l'exposition aux champs électromagnétiques. À ce jour, il n'existe pas d'indicateurs biologiques spécifiques permettant d'établir un tableau clinique typique. Des troubles similaires sont décrits dans les cas de l'hypersensibilité chimique, du syndrome du bâtiment malsain, ou encore du syndrome de la guerre du Golfe, pour lesquels aucune relation causale n'a pu être établie avec l'agent responsable désigné (lorsqu'il y en a un), ni avec aucun autre agent environnemental. L'OMS englobe ce syndrome EHS sous le terme général d'intolérance environnementale idiopathique (IEI), soulignant son absence de spécificité à un agent.

Ce syndrome concerne un très faible pourcentage de la population $(<3 \%)$, dont une majorité de femmes qui prétendent, pour la plupart, être en mesure de détecter la présence d'un champ électromagnétique. Toutefois, aucun système sensoriel humain permettant de percevoir les champs électromagnétiques aux niveaux d'exposition rencontrés dans la vie courante, n'a pu être identifié. Par ailleurs, plus d'une trentaine d'études ont été publiées dans lesquelles des individus EHS ont été exposés à des champs similaires à ceux auxquels ils attribuaient leurs symptômes (Rubin et al., 2010). L'objectif de ces études était de provoquer l'apparition des symptômes en conditions contrôlées de laboratoire par des essais appelés de « tests de provocation ». Les études menées en double aveugle - c'est-à-dire que ni la personne volontaire, ni le médecin examinateur ne savent si le champ est présent ou non - ont montré que les symptômes n'étaient pas corrélés à la présence du champ. De plus, ni les personnes se plaignant d'EHS, ni celles ne s'en plaignant pas, ne sont capables de détecter la présence du champ magnétique. En revanche, le fait de se savoir exposé entraîne l'apparition des symptômes chez les sujets du 
groupe EHS, en l'absence de champ électromagnétique (exposition factice), ce qui ne se produit pas dans le groupe d'individus témoin (non EHS). Quelle qu'en soit la cause, il est certain que l'EHS entraîne des souffrances et peut affecter la qualité de vie des personnes atteintes qui ne sont parfois plus en mesure, pour les cas les plus extrêmes, de mener une vie sociale normale.

Ce type de syndrome questionne donc les relations entre environnement, information sur les risques et santé. La notion d'exposition sociocognitive permet de rechercher les liens éventuels entre information et santé pour prendre en considération, par exemple, les effets liés à la présence d'antennes relais dans le voisinage quand ces dernières sont stigmatisées comme potentiellement porteuses d'effets néfastes pour la santé. Comme l'a mentionné L. Challis ${ }^{7}$ pendant son audition pour l'expertise de l'Afsset (2009), les observations de médecins généralistes au Royaume-Uni sont importantes et doivent être prises en compte en ce qu'elles font état d'une association entre la peur des antennes relais et la production de symptômes : "Dans les cinq différentes villes invitées à participer (...), $90 \%$ des préoccupations portaient sur les antennes relais. Nous avons parlé avec les médecins et ils ont dit que la peur des antennes relais produit des symptômes. Ce n'est donc pas quelque chose que vous pouvez écarter».

Bien que reposant sur l'expérience clinique de médecins, l'évaluation demeure imprécise et l'effet, signalant un possible impact sanitaire, doit être considéré comme hypothétique. En tant que «signal » méritant d'être étudié, cet effet pose la question des mécanismes auxquels associer la production des symptômes dans ce contexte. Nous proposons de les répartir en trois catégories distinctes : l'effet nocebo, le stress, la règle de symétrie.

L'effet nocebo représente le versant négatif de l'effet placebo, décrit initialement quand un médicament ou un geste médical, en principe neutre, produit des symptômes chez la personne. Cet effet étant situé dans la relation de soin avec les croyances (en l'occurrence la croyance d'un effet indésirable pour la santé) que cette relation peut nourrir, il ne paraît pas pour ces raisons totalement approprié à l'exposition aux antennes relais puisque celle-ci n'entre pas dans le cadre d'une relation de soin. Toutefois, l'étude du syndrome d'électro hypersensibilité (EHS) recourt à ce concept (Rubin et al., 2010).

Apparu dans les années 1930 avec les travaux de Selye (1975), le concept de stress trouverait à s'appliquer ici dans la mesure où les antennes relais, avec les

\footnotetext{
7 Lawrie Challis est membre du groupe d'experts indépendants du MTHR (Mobile Telecommunications and Health Research Programme) au Royaume-Uni et dont le rapport d'expertise est disponible en ligne: http://www.mthr.org.uk/documents/MTHR_report_2007.pdf
} 
informations préoccupantes et les controverses les concernant, constitueraient un stresseur chronique dans l'environnement des personnes; divers symptômes de stress pourraient alors être associés à cette cause. On notera que les experts sont depuis longtemps attentifs à cette possibilité de stress, celle-ci étant implicite quand apparut dès la fin des années 1950 l'enjeu de «la présentation d'informations susceptibles de générer de l'anxiété, compte tenu de la capacité des populations à les endurer », lors d'une des premières expertises collectives - sinon la première - intégrant l'information dans l'évaluation des risques (WHO, 1958).

En contraste avec ces concepts relativement connus, la démonstration du lien entre la cognition (dans le sens large d'une information sur des risques reçue et traitée par les individus) et la santé, est plus récente. Elle se trouve dans quelques études reposant sur la règle de symétrie (Brownlee et al., 2000), que nous présentons brièvement à l'aide d'un cas. Ces études concluent en effet sur l'existence d'un lien et donnent comme exemple une controverse aux Etats-Unis concernant le maïs transgénique. Une association de consommateurs signale que des traces de maïs transgénique ont été trouvées dans des produits alimentaires comme les taco shells, alors que les industriels maintiennent que le maïs transgénique est utilisé uniquement pour nourrir le bétail. La nouvelle de ces traces attire l'attention des médias et du public et se diffuse rapidement. Le Center for Desease Control (CDC) reçoit les cas de 51 personnes présentant des symptômes (modérés : état de faiblesse, vertiges, ou graves : évanouissement, hospitalisation) qu'elles attribuent à leur exposition alimentaire au maïs. Toutefois, une investigation complète des cas par le CDC, s'appuyant sur des entretiens approfondis et des tests sérologiques, a conclu qu'aucune de ces personnes n'avait été exposée au maïs transgénique, et qu'une réaction allergique liée à l'exposition ne pouvait expliquer les symptômes signalés.

Deux explications, qui ne sont pas mutuellement exclusives, sont alors recherchées pour s'appliquer à ce phénomène qui correspond, dans notre approche, aux effets d'une exposition sociocognitive. La première propose que les personnes qui avaient au préalable un symptôme inexpliqué ont pu trouver une explication utile avec l'information concernant la présence de maïs transgénique dans la nourriture. Cette situation apparait aussi sous l'appellation « d'opportunisme cognitif »(Bronner et Géhin, 2010), que les auteurs décrivent comme une situation où l'individu, souffrant déjà de symptômes pour lesquels il n'a pas d'explication satisfaisante, trouve ainsi une cause dès lors qu'il peut en concevoir une dans son environnement (comme avec le cas des radiofréquences émises par les antennes ou le téléphone mobile). Selon la seconde explication, les informations ont pu susciter chez ceux qui croyaient avoir consommé du maïs transgénique, et qui se sont ainsi considérés « exposés », une vigilance accrue aux symptômes allergiques qu'ils n'auraient pas remarqués sans cela. 
Ces deux explications théoriques aboutissent à la règle de symétrie (symmetry rule) selon laquelle les cognitions se rapportant à la maladie reposent sur la dualité des représentations en mémoire : l'une concrète, l'autre abstraite. La construction mentale d'un état de santé inclut une représentation abstraite (par exemple, avoir consommé des aliments contenant du maïs transgénique) et des symptômes concrets (par exemple, nausée, faiblesse), sachant que la présence de l'une des représentations entraînera l'autre. Ainsi, « les personnes chercheront et trouveront des représentations pour expliquer leurs symptômes, et chercheront et trouveront des symptômes pour rendre concrètes leurs représentation de la maladie » (Brewer et al., 2008).

Au-delà de la seule analogie, la transposition de la règle de symétrie aux symptômes associés à une exposition à la téléphonie mobile demanderait un dispositif de recherche spécifique. Il serait par exemple nécessaire de préciser quelle part est attribuable à l'exposition visuelle aux antennes relais, et quelle part est attribuable à l'exposition cognitive, cette dernière renvoyant aux informations potentiellement préoccupantes reçues sur les risques associés aux radiofréquences. L'intégration des effets de l'exposition sociocognitive pourrait se concevoir également dans les recherches portant sur les risques sanitaires des radiofréquences dès lors qu'elles reposent sur des déclarations d'usage ou, plus largement, de données issues d'entretiens. Ainsi certaines des limites rencontrées par l'étude Interphone dont les résultats ont récemment été publiés (Cardis et al., 2010), comme par exemple des valeurs non plausibles d'utilisation déclarée du téléphone mobile, ou un risque de gliome accru en Suède mais pas au Royaume-Uni, pourraient être analysées parmi les effets de l'exposition sociocognitive, laquelle peut affecter les déclarations d'utilisation et varier vraisemblablement d'un pays à un autre et dans le temps.

Une autre dimension collective de l'exposition à l'information demanderait aussi à être évaluée et intégrée : en situation de controverse sur les risques, quels sont les effets des conseils de prudence ? Dans le domaine de la téléphonie mobile, les mesures de précaution sont parfois invoquées comme moyen pour répondre aux préoccupations des populations, cependant que certaines études retiennent comme hypothèse que cette approche pourrait causer ou exacerber lesdites préoccupations. Une étude expérimentale conduite en Allemagne permet aux auteurs de conclure que les mesures de précaution pourraient déclencher des préoccupations et amplifier les perceptions de risques (Wiedemann et Schütz, 2005). Pour vérifier ce qu'il en est au Royaume-Uni de l'impact des avis diffusés par le gouvernement concernant les risques potentiels de la téléphonie mobile, les chercheurs utilisent la méthode $\mathrm{du}$ focus group (en l'occurrence des groupes présentant des caractéristiques différentes en âge, sensibilisation et préoccupations aux risque des antennes relais, usages du téléphonie mobile). Les résultats montrent que la compréhension des avis 
n'apparaît pas principalement ancrée dans la précaution, l'avis paraissant généralement interprété comme une information préoccupante plutôt que rassurante (Barnett et al., 2007). Une étude conduite récemment sur un échantillon de 1687 personnes au Danemark et s'appliquant à la téléphonie mobile, montre que l'information peut augmenter les préoccupations parmi une proportion importante de la population (Nielsen et al., 2010). Obtenus par des méthodologies différentes et dans plusieurs pays, ces résultats convergent pour montrer le rôle actif des populations dans leur interprétation de l'information sur les risques.

\section{Conclusion}

Alors que la période précédente était principalement marquée depuis le début des années 1970 par les difficultés pour identifier et dire le risque quand il est avéré dans divers domaines, une ère nouvelle à laquelle semblent participer les radiofréquences se caractérise simultanément par une grande attention (publique et scientifique) se traduisant par de nombreuses études et par des résultats négatifs. Peau de chagrin inversée, la téléphonie mobile semble s'étendre à mesure que s'exprime notre irrépressible désir de communication et d'ubiquité. Et comme pour la mystérieuse peau d'onagre, les sciences et techniques paraissent impuissantes : les tourments et souffrances qu'une partie de la population attribue à la téléphonie mobile, alors qu'elle exauce par ailleurs nos vœux, ne trouvent guère d'explication plausible et susceptible de ralentir son extension apparemment inéluctable.

Face aux symptômes attribués aux radiofréquences et, plus généralement, compte tenu des préoccupations de la population quant à la possibilité d'un risque, les gouvernants et les chercheurs ne peuvent rester insensibles et les évaluations de risques se justifient d'autant plus que la quasi-totalité de la population se trouve exposée. Plusieurs catégories d'exposition sont donc considérées. Ainsi les populations ne sont-elles pas seulement exposées à l'agent physique que constituent les radiofréquences, elles sont également exposées à la téléphonie mobile en tant qu'objet social dont la fréquentation s'est rapidement imposée et généralisée, laissant peu de temps d'adaptation à cette innovation technologique et aux changements qu'elle induit. Un autre niveau d'exposition à prendre en compte est celui de l'information provenant des nombreuses études et expertises consacrées à la recherche d'effets biologiques et sanitaires, ainsi que celui de l'information médiatique portant sur les symptômes et phénomènes divers attribués aux radiofréquences. Ces différents niveaux d'exposition, que nous regroupons sous le terme d'exposition sociocognitive, permettent de considérer les liens entre information sur les risques et santé, et plusieurs mécanismes possibles sont identifiés : l'effet nocebo, le stress, et la règle de symétrie. En corollaire à cette approche, un enjeu à considérer sera celui de la gestion de l'incertitude, 
laquelle se traduit généralement par des mesures de protection des populations les plus vulnérables a priori à l'agent physique (femmes enceintes, enfants, personnes malades). Or, dans la perspective de l'exposition sociocognitive, il est possible que d'autres populations soient vulnérables et les mesures de protection, comme les situations extrêmes, seraient alors à définir. Pour leur intégration concrète à l'évaluation des risques, les concepts explorés ici demanderaient à être approfondis en expologie et par des recherches interdisciplinaires, surtout en ce qui concerne les dimensions individuelles et collectives des liens entre environnement, information sur les risques et santé.

\section{RÉFÉRENCES}

Afsset (2009) Mise à jour de l'expertise relative aux radiofréquences. Saisine 2007/007. Octobre 2009. Afsset, Maisons Alfort. Consultable en ligne, 18 septembre 2010: www.afsset.fr/upload/.../Rapport_RF_20_151009_1.pdf.

Amalberti R. (2009) L'impact des nouvelles technologies sur le risque, Revue de l'ASN, $\mathrm{n}^{\circ} 185$, décembre 2009.

Barnett J., Timotijevic L., Shepherd R. (2007) Public responses to precautionary information from the Department of Health (UK) about possible health risks from mobile phones, Health Policy 82, 240-250.

Brewer N.T., Hallman W.K., Kipen H.M. (2008) The symmetry rule: a seven-year study of symptoms and explanatory labels among Gulf War veterans, Risk Analysis 28, 1737-1748.

Bronner G., Géhin E. (2010) L'inquiétant principe de précaution. PUF, Paris.

Brownlee S., Leventhal H., Leventhal E.A. (2000) Regulation, self-regulation, and construction of the self in the maintenance of physical health. In: Handbook of Self-Regulation. Research, theory and applications (M. Boekartz, P.R. Pintrick, M. Zeidner, Eds.) pp. 369-393. Elsevier Academy Press, San Diego.

Cardis E. et al. (2010) Brain tumor risk in relation to mobile phone use: results of the INTERPHONE international case control study, Int. J. Epidemiol. 39, http:ije.oxfordjournals.org (17 mai 2010).

Dab W. (2010) Les syndromes, médicalement inexpliqués attribués à l'environnement : un révélateur de la relation entre l'environnement et la santé, Sciences Sociales et Santé 28, 35-40.

Hubert P. (2000) Approche comparée de quelques évolutions de la gestion du risque en France à travers l'organisation des Agences. Dans : Séminaire Risques collectifs et situations de crise, 27 avril 2000, École des Mines de Paris, séance 17 (C. Gilbert, Ed.) pp. 53-78. CNRS, Grenoble.

ICNIRP (1998) Guidelines for limiting exposure to time-varying electric, magnetic and electromagnetic fields (up to $300 \mathrm{GHz}$ ), Health Phys. 74, 494-522.

Nielsen J.B., Elstein A., Gyrd-Hansen D., Kildemoes H.W., Kristiansen I.S., Stovring H. (2010) Effects of Alternative Styles of Risk Information on EMF Risk Perception, Bioelectromagnetics 31, 504-512.

OCDE (2008) L'OCDE en chiffres - Édition 2008. Paris : Les éditions de l'OCDE.

Pidgeon N., Kasperson R., Slovic P. (2003) The social amplification of risk. Cambridge University Press, Cambridge.

Poortinga W., Cox P., Pidgeon N. (2008) The Perceived Health Risks of Indoor Radon Gas and Overhead Powerlines: A Comparative Multilevel Approach, Risk Analysis 28, 235-248.

Renn O. (2008) Risk governance: Coping with uncertainty in a complex world. Earthscan Publication Ltd, London. 
Rubin J.G., Nieto-Hernandez R., Wessely S. (2010) Idiopathic Environmental Intolerance attributed to electromagnetic fields (formerly "electromagnetic hypersensitivity"): An updated systematic review of provocation studies, Bioelectromagnetics 31, 1-11.

Selye H. (1975) Le stress de la vie. $2^{\mathrm{e}}$ édn. Gallimard, Paris.

Slovic P. (2000) The perception of risk. Earthscan Publication Ltd, London.

Veyret B. (2010) Les communications sans fil et les radiofréquences, Dans : Champs électromagnétiques, environnement et santé (A. Perrin et M. Soucques, Eds.) pp. 69-86. Verlag, Paris.

Wiedemann P.M., Schütz H. (2005) The precautionary principle and risk perception: experimental studies in the EMF area, Environm. Health Persp. 113, 402-405

WHO (1958) Mental Health Aspects of the Peaceful Uses of Atomic Energy. Report of a Study Group. Technical Report Series $N^{\circ} 151$. World Health Organization, Genève. 